\title{
Design Optimization of Stress Relief Grooves in Lever Guide of Pressure Vessel for Food Processing
}

\author{
Yuichi Otsuka $^{1 *}$, Hamirdin Bin Baron ${ }^{2}$, Yoshiharu Mutoh ${ }^{3}$ \\ ${ }^{1}$ Top Runner Incubation Center for Academia-Industry Fusion, Nagaoka University of Technology, \\ Niigata, Japan \\ ${ }^{2}$ Undergraduate School of Mechanical Engineering, Nagaoka University of Technology, \\ Niigata, Japan \\ ${ }^{3}$ Department of System Safety, Nagaoka University of Technology, Niigata, Japan \\ Email: *otsuka@vos.nagaokaut.ac.jp
}

Received November 20, 2011; revised February 7, 2012; accepted February 15, 2012

\begin{abstract}
A stress relief groove is introduced in the $\mathrm{R}$ area and the stress is analyzed using a finite element method (FEM). Then the relief of the stress concentration in the vicinity of the pressure vessel is measured based on these results. When designing a stress relief groove, the lever must overhang the groove $(L>0)$. By introducing a stress relief groove to the $\mathrm{R}$ area, maximum stress on the lever guide can be reduced by $10 \%$. This enables the reduction of the maximum stress (Mises stress) to be less than the fatigue strength. Furthermore, the location where maximum stress occurs on the lever guide changes in accordance with the clearance between the lever and lever guide. This identified the need to take into account the deviation factor such as design clearance in modeling process.
\end{abstract}

Keywords: Stress Relief Groove; Stress Concentration Factor; Finite Element Analysis; Pressure Vessel

\section{Introduction}

From the late 1980s, in preparation for the utilization of high-pressure processing in the Japanese domestic food industry, high-pressure processing was first utilized in the commercialization of jam and fruit juice drinks. Today, high-pressure food has become an increasingly familiar product as a result of the commercialization of highlyfunctional foods such as cooked rice in aseptic packaging and pressure-steamed brown rice. Research is currently being conducted with regards to the use of high-pressure treatment as a food-processing method, with the aim of improving the productivity and quality, as well as adding a hypoallergenic quality [1].

Experiments have proven that there are various benefits at pressures higher than $100 \mathrm{MPa}$ (called ultra-high pressure in many cases); however, the cost of ultra-high pressure processing equipment is high and its production is small. Moreover, because operating the ultra-high pressure processing equipment requires specialized knowledge or skills, such small-scale equipment for everyday use is not yet widely available. In addition, the development of new equipment requires advanced technical capabilities and a definitive plan, and there are many other complications such as securing an appropriate sales chan-

${ }^{*}$ Corresponding author. nel as well as obtaining financial investment for high developmental and manufacturing costs.

With the conventional seal mechanism for ultra-high pressure equipment, a push-type structure for the cover, similar to that for a press, was used [1]. When this method is used, the structure of the vessel and lid is simple, and the structure is easily able to withstand ultra-high pressure. However, the cost of a press mechanism is high and its installation increases the overall size of the equipment. In order to reduce the cost and size, the seal method shown in Figures 1 and $\mathbf{2}$ was proposed. This consists of a cylindrical vessel with a cover at each end fitted with a gasket to maintain the pressure and the cover is closed by a lever, which ensures that a firm seal is established. In the upper part of the vessel, the lever is inserted into the lever guide that has been processed using a wire cut (EDM).

Using this structure, a concentration of stress [2] occurs in the vicinity of the area $\mathrm{R}$ because of the contact between the lever and lever guide. The stress concentrations at the $\mathrm{R}$ area of the lever guide will easily become the origin of fracture. Therefore, it is vital to implement a design that eases the stress in the vicinity of the $\mathrm{R}$ area.

In this study, a stress relief groove [3] is introduced in the $\mathrm{R}$ area, as shown in Figure 3. The stress is subsequently analyzed using a finite element method (FEM) [4]. Then the relief of the stress concentration in the vicinity of the pressure vessel is measured based on these results. 


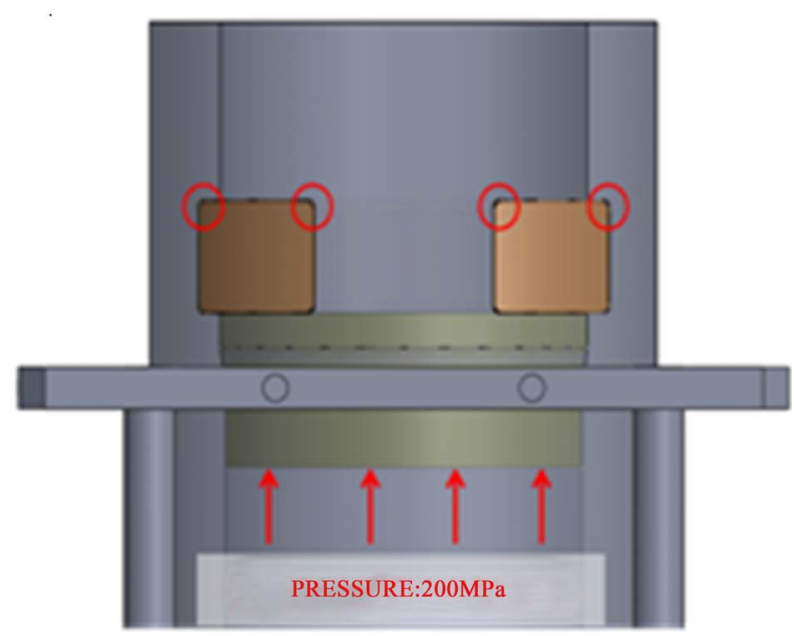

Figure 1. Pin-arm sealed pressure vessel structure for food processing.

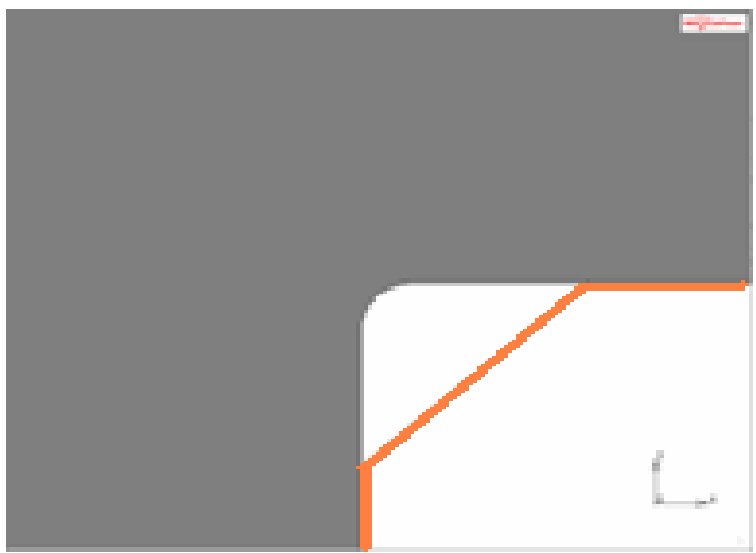

(a)

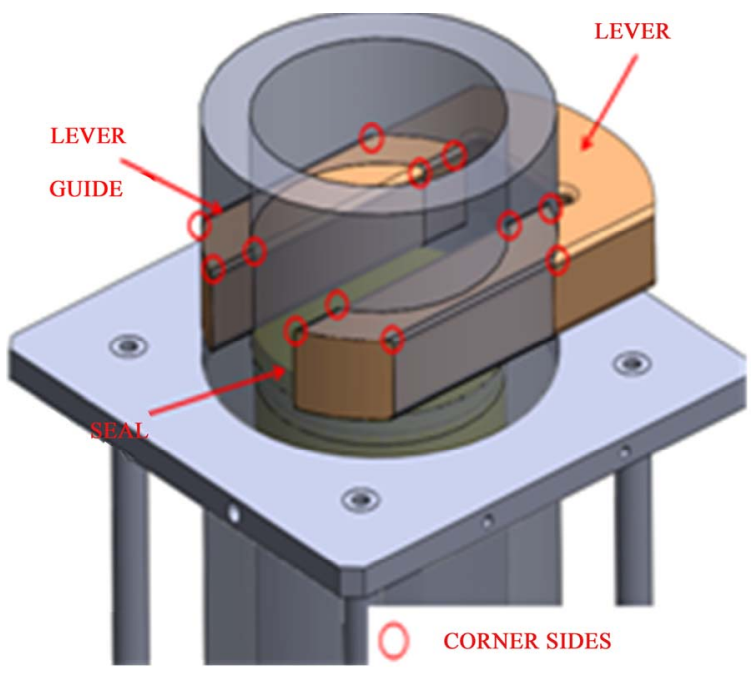

Figure 2. Upper view of pressure vessel.

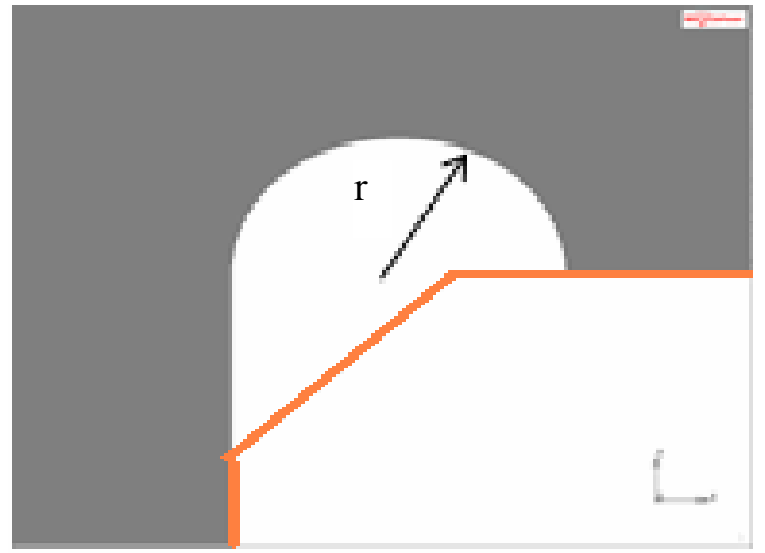

(b)

Figure 3. Conventional (a) and with stress relief groove design (b) at the corner side of lever guide. (a) Without groove; (b) With groove.

\section{Examination of the Optimal Groove Shape Using 2-D Analysis}

In order to select an optimal shape for the stress relief groove, a simplified 2-D model of the pressure vessel was created and analysis was carried out using this model.

\subsection{Examination of the Optimal Groove Shape Using 2-D Analysis}

The commonly used FEM program MARC/MENTAT was used in this analysis. We analyzed the data using 2-D elasticity, and three contact point stress elements were used.

Figures 4-6 show the boundary parameters and FEM model used in this analysis. The FEM model was cut in half longitudinally to obtain a cross-section and four models were created. One was a conventional shape without a groove, and the other three had semicircular grooves of radii 1,2 and $3 \mathrm{~mm}$, respectively. The friction between the lever guide and lever was defined, and the Lagrange friction algorithm was used to define the friction behavior. Table 1 shows the values for the properties of the material used in the analysis model. To simulate a highpressure during the operation of the equipment, a step-shaped moving velocity, $V_{y}=0.35 \mathrm{~m} / \mathrm{s}$, was provided to the rigid lever in the $y$ direction. This moving velocity is an assumed value when the pressure is increased to $200 \mathrm{MPa}$.

\subsection{2-D Analysis Results}

\subsubsection{Mises Stress and Maximum Principal Stress Distribution}

Figure 7 shows the Mises stress distribution and maximum principal stress distribution in the vicinity of the $\mathrm{R}$ area for the conventional model without a groove and for the models with a groove of radii $=1,2$ and $3 \mathrm{~mm}$. The predicted stress concentration points $A(R$ area) and $B$ (lever contact area) are shown in Table 2. 


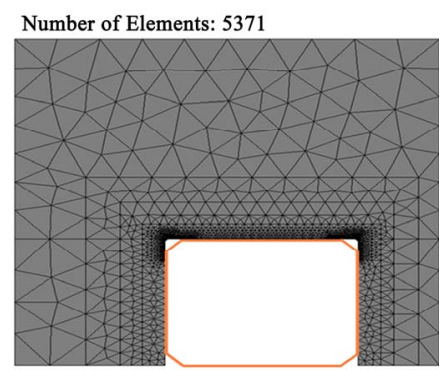

Figure 4. 2-dimentional FEM model of lever guide. The model is half of the upper side because of its symmetric structure.

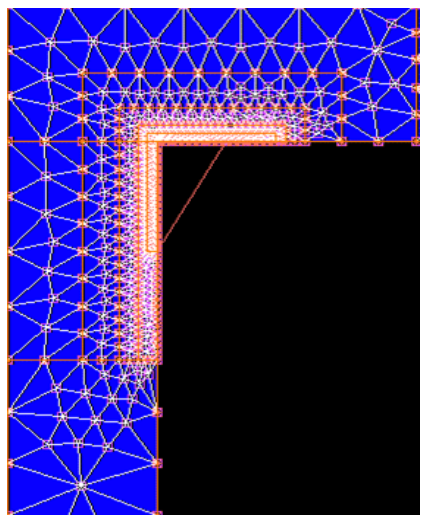

(a)

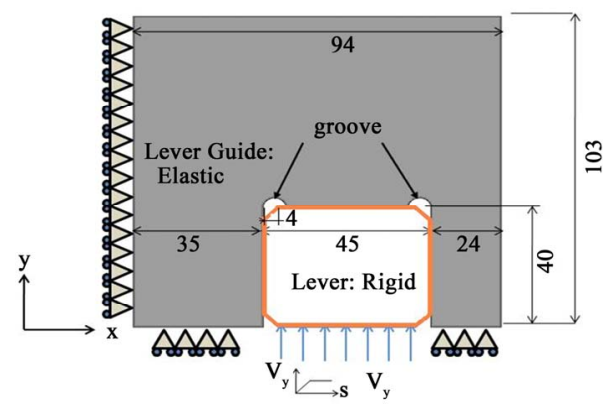

Figure 5. Boundary conditions of the FEM model 1.

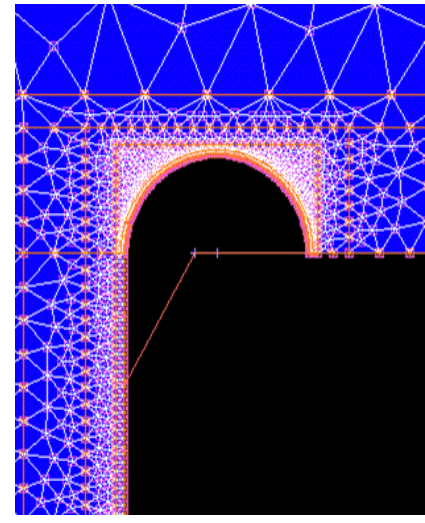

(b)

Figure 6. Detailed meshing conditions around the root of corner or groove. (a) Without groove; (b) With groove a ( $r=3 \mathrm{~mm})$.

Table 1. Material properties of JIS SUS630 used for the lever guide.

\begin{tabular}{ccccc}
\hline & Young's Modulus & Poisson's Ratio & Density & Friction Coefficient \\
\hline JIS SUS630 & $205 \mathrm{GPa}$ & 0.3 & $7800 \mathrm{~kg} / \mathrm{m}^{3}$ & 0.2 \\
\hline
\end{tabular}

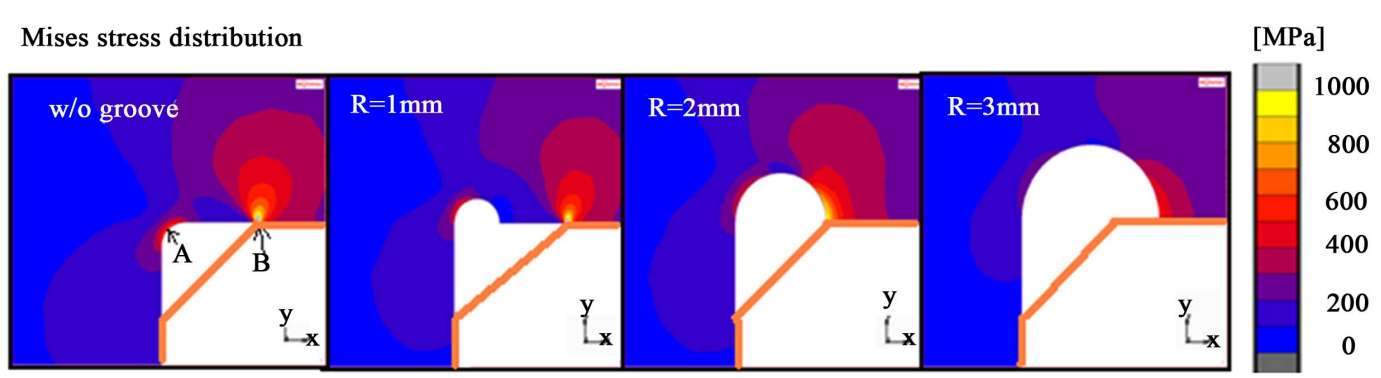

(a)

Max principal stress distribution

[MPa]

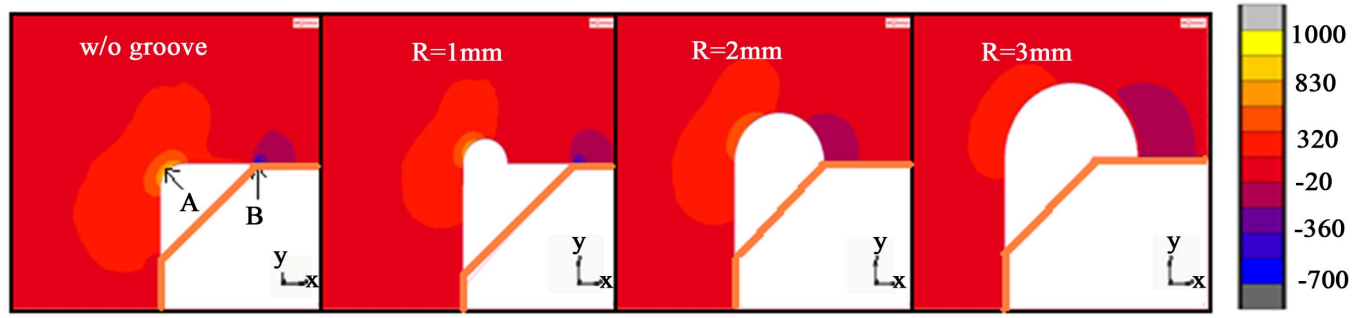

(b)

Figure 7. Effect of radius $\mathbf{r}$ for stress concentrating conditions around the contact point. (a) Mises stress distributions (relating to plastic deformation); (b) Max principal stress distributions (relating to brittle fracture). 
According to the stress distribution in Figure 7, the maximum stress that occurs on the lever guide decreases in a model having a stress relief groove as compared with the one without the groove. The degree of reduction of maximum stresses becomes higher according to the increase of the groove radius from 1 to $3 \mathrm{~mm}$.

Table 2 shows that the Mises stress at points A and B and the maximum principal stress at point $\mathrm{A}$ in the model with a $3 \mathrm{~mm}$ groove radius. The maximum stress becomes $60 \%$ less than that of the model without a groove. The maximum principle stress at point B in the models with 2 $\mathrm{mm}$ and $3 \mathrm{~mm}$ groove radii are approximately $90 \%$ less than that of the conventional model and/or the model with a $1 \mathrm{~mm}$ groove radius. The results indicate that the location of the lever contact edges have a significant effect on the stress on the inner surface of the lever guide.

Table 3 shows the result of maximum stresses at the root of grooves in the cases of eclipse shape. Interestingly, stresses show their lowest values in the cases of $t$ $=b$, which means the groove shape is half circle. The reason is not clear. Probably the changes in contacting area between the lever guides and the lever can affect the stress concentration conditions.

\subsubsection{Relation between Overhang Range $L$ and Stress Distribution}

To examine the relation between the overhang range $L$ and the stress distribution, we created models having distances from the lever edge to the lever contact edge (overhang) $L$ of $4,2,0$ and $-2 \mathrm{~mm}$, and analyzed the stress data. The results of the analyses for the Mises stress and maximum stress distribution are shown in Figure 8. The results show that there is no significant change in the magnitude of stress at both point $\mathrm{A}$ and point $\mathrm{B}$ for the models with $L=0,2$ and $4 \mathrm{~mm}$, but for the model with $L$ $=-2 \mathrm{~mm}$, there is concentrated compressive stress. We believe that when the overhang is $L<0$, the stress is concentrated at the contact edge, and thus the concentrated compressive stress is seen only in the model with $L$ $=-2 \mathrm{~mm}$.
Table 2. Mises and max principal stress at point $A$ and $B$; point $A$ is the root of the corner and point $B$ is the edge of contact regions.

\begin{tabular}{ccccc}
\hline & \multicolumn{2}{c}{ Mises Stress [MPa] } & \multicolumn{2}{c}{ Max Principal Stress [MPa] } \\
\cline { 2 - 5 } Model & $\mathrm{A}$ & $\mathrm{B}$ & & $\mathrm{B}$ \\
\hline Without groove & 667 & 1742 & 788 & -705 \\
Groove, $r=1 \mathrm{~mm}$ & 508 & 1614 & 621 & -664 \\
Groove, $r=2 \mathrm{~mm}$ & 426 & 960 & 507 & -37 \\
Groove, $r=3 \mathrm{~mm}$ & 255 & 526 & 302 & -17 \\
\hline
\end{tabular}

Table 3. Mises and max principal stress in the cases of eclipse shape grooves.

\begin{tabular}{|c|c|c|c|}
\hline \multicolumn{2}{|c|}{ Shape of eclipse $t$; depth, $2 b$; diameter } & \multicolumn{2}{|c|}{ Maxinum stress $[\mathrm{MPa}]$} \\
\hline$t$ & $2 b$ & Mises Stress & Max Principal \\
\hline 1 & 2 & 539 & 473 \\
\hline 1 & 1 & 766 & 601 \\
\hline 0.5 & 2 & 592 & 656 \\
\hline 2 & 4 & 667 & 332 \\
\hline 2 & 2 & 507 & 426 \\
\hline 1 & 4 & 1011 & 475 \\
\hline 3 & 6 & 557 & 262 \\
\hline 3 & 3 & 513 & 350 \\
\hline 1.5 & 6 & 869 & 363 \\
\hline
\end{tabular}

Max principal stress distribution

[MPa]
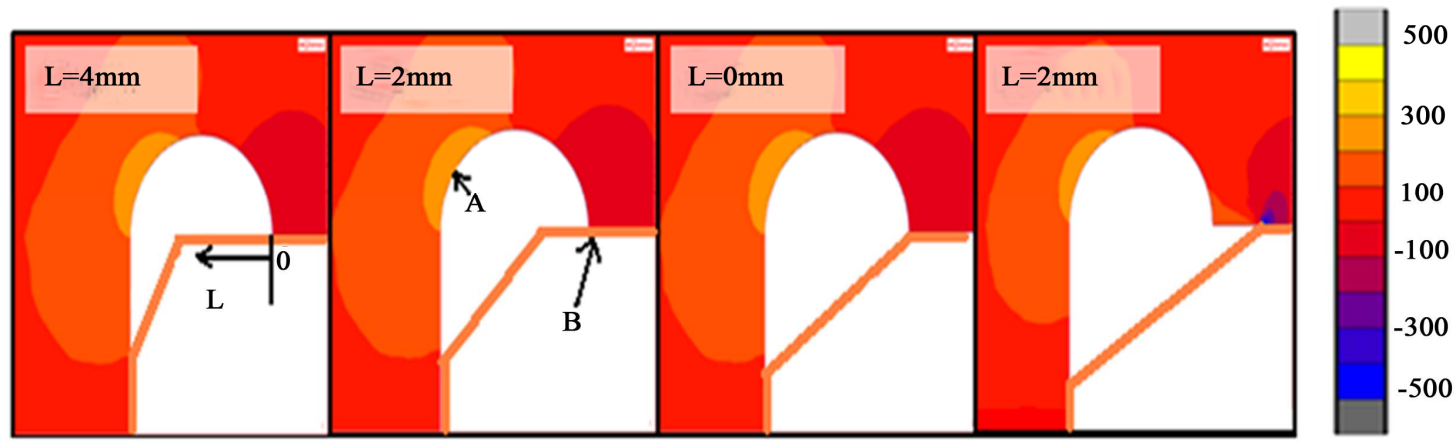

Figure 8. Effect of over-hang in contact region for max principal stresses in the cases of $L=4,2,0,-2$ models. 
Therefore, when selecting the profile of the stress relief groove, the overhang $L$ has to be greater than 0 in order to reduce the compressive stress on the inner surface of the lever guide, and thus we chose a stress relief groove with radius $r=3 \mathrm{~mm}(L=0)$.

\section{3-D Analysis}

We performed 3-D analysis to measure the stress at the six locations where stress concentration is predicted during the pressurization of the pressure vessel as shown in Figure 9: points F, H, E, and C on the inner side of the lever guide; and points $\mathrm{G}$ and $\mathrm{D}$ on the outer side of the lever guide.

\subsection{3-D Analysis Method and Model}

Stress analysis was performed for Case 1 and Case 2 below, as shown in Figures 10 and 11, respectively.

\section{1) Case 1}

Model 1 without a groove and Model 2 with a 3-mm radius groove, which was selected based on 2-D analysis were compared. The stress concentration relief obtained by using the stress relief groove was investigated. The maximum tolerance for the machining dimensions between the lever and lever guide was $0.6 \mathrm{~mm}$. In Case 1, a fit tolerance of $0.3 \mathrm{~mm}$ was set for the inner side and outer side of each lever guide, which means that the lever was inserted into the center of the lever guide space.

2) Case 2

In Case 2, stress analysis was performed on the $\mathrm{R}$ area when, using the dimensional tolerance of the lever, the lever made contact with the surface of the outer side of the lever guide with a clearance of $0.6 \mathrm{~mm}$ from surface of the inner side (model 3) and when the lever made contact with the surface of the inner side of the lever guide with a clearance of $0.6 \mathrm{~mm}$ from the surface of the outer side (model 4). Model 3 and model 4 are shown in Figure 11.

The dimensions of the 3-D model are shown in Figure 9. The analysis was performed using 3-D elasticity, and four contact point stress elements were used, as shown in Figure 9. Figure 12 shows the model and boundary parameters for the 3-D analysis. The friction conditions and the values for the material properties were the same as those for the 2-D analysis. The numbers of elements are as the follows; Model $1=103,389$, Model $2=102,706$, Model $3=103,528$, Model $4=103,528$, respectively.

\subsection{3-D Analysis Results}

\subsubsection{Case 1: Effect of Stress Concentration Relief by the Groove}

Figure 13 shows the Mises stress distribution for model 1 without a groove in Case 1. The stress values for points C, D, E, F, G, and H are shown in Table 4.

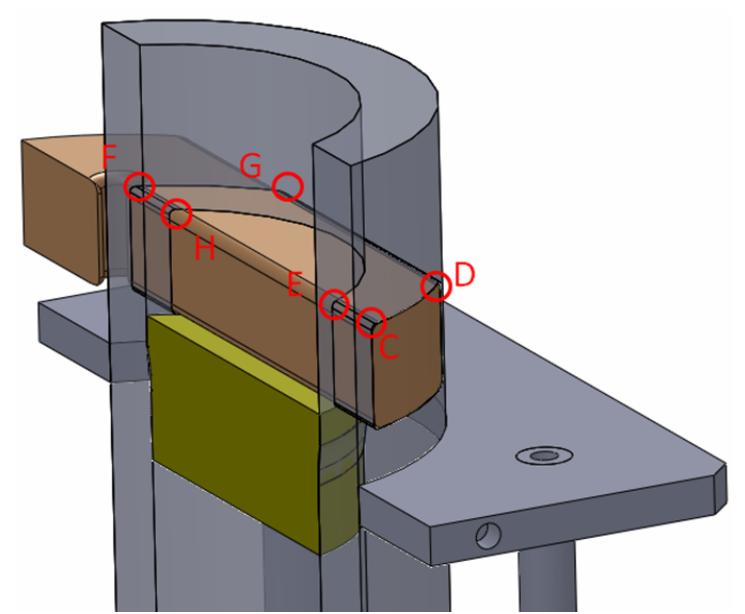

Figure 9. 3-dimensional finite element model of pressure vessel. Red circles show possible initial failure point.
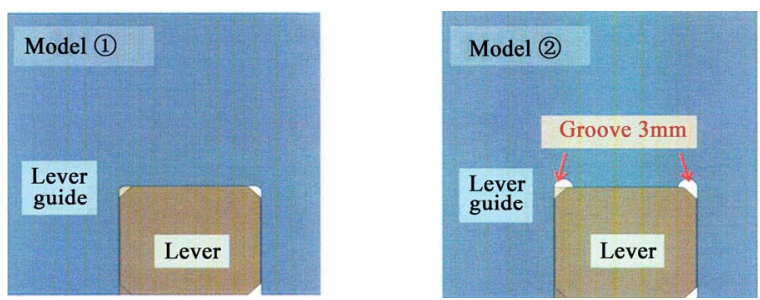

Figure 10. Models for case 1. Conventional model (model 1) and with stress relief groove $3 \mathrm{~mm}$ model (model 2).
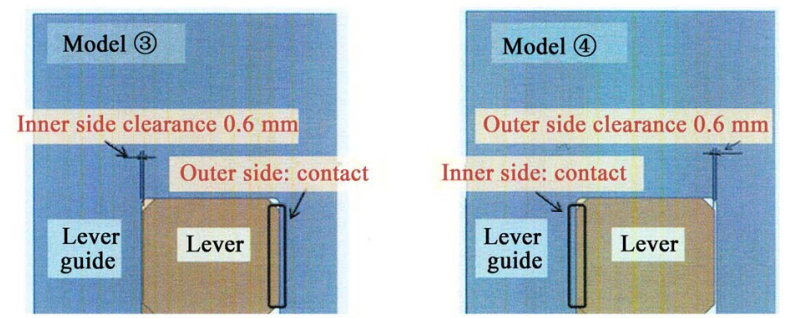

Figure 11. Models for case 2. Clearance $0.6 \mathrm{~mm}$ at outer side (model 3) and inner side (model 4) of the vessel.
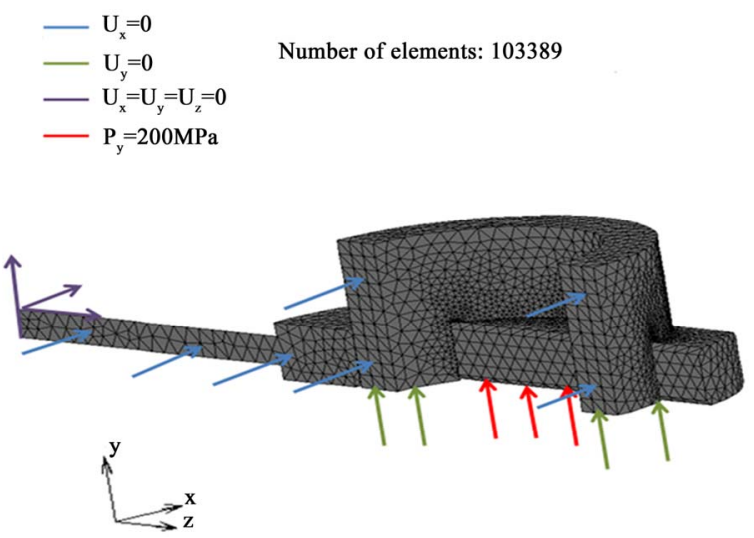

Figure 12. Meshing and boundary conditions of FE models. Number of elements; Model $1=103,389$, Model $2=102,706$, Modle $3=$ 103,528, Model $4=103,528$. 


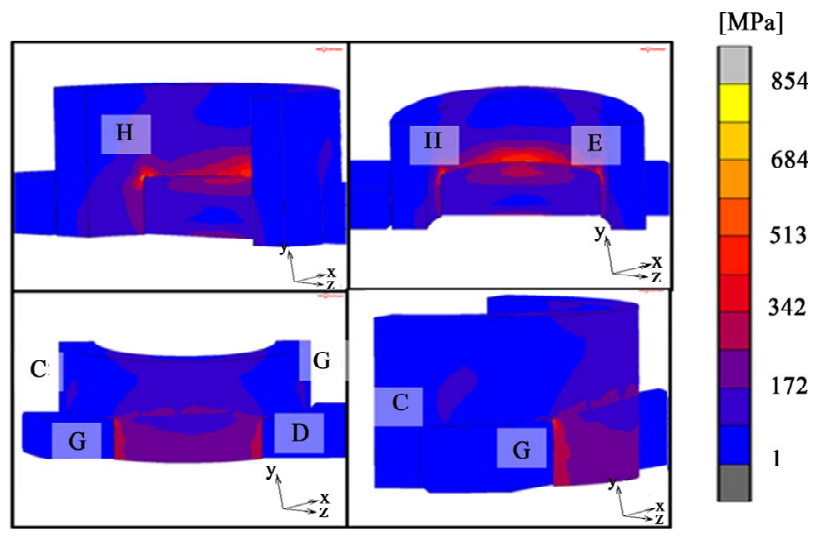

Figure 13. Mises stress distribution of Model 1.

Table 4. Stress analysis result for case 1; effect of stress relief grooves for reducing maximum stresses.

\begin{tabular}{ccccc}
\hline & \multicolumn{4}{c}{ Maximum stress [MPa] } \\
\cline { 2 - 5 } Point & \multicolumn{2}{c}{ Model 1 } & \multicolumn{2}{c}{ Model 2 } \\
& (without groove) & (with groove $r=3 \mathrm{~mm}$ ) \\
\cline { 2 - 5 } & Mises & Max Principal & Mises & Max Principal \\
\hline C & 105 & 91 & 108 & 103 \\
D & 332 & 263 & 433 & 413 \\
E & 773 & 801 & 670 & 512 \\
F & 105 & 94 & 109 & 103 \\
G & 249 & 192 & 286 & 302 \\
H & 744 & 704 & 561 & 627 \\
\hline
\end{tabular}

When the vessel is pressurized, the lever is subjected to a bending moment and because the $\mathrm{R}$ area for point $\mathrm{E}$ and point $\mathrm{H}$ acts as a fulcrum for a lever-bending deformation, it is evident from Figure 13 and Table 4 that the stress is greater at points $\mathrm{E}$ and $\mathrm{H}$ than at points $\mathrm{C}, \mathrm{D}, \mathrm{F}$, and G. If model 1 and model 2 are compared, the stress on the $\mathrm{R}$ area is reduced by approximately $10 \%$ by introducing a groove with a $3-\mathrm{mm}$ radius to the $\mathrm{R}$ area at points $\mathrm{E}$ and $\mathrm{H}$. The fatigue strength of this material is $700 \mathrm{MPa}$ [5], and therefore the durability can be expected to be $10^{7}$ cycles.

\subsubsection{Case 2: Relation between Clearance and Maximum Stress Locations}

The Mises stress distribution and the maximum principal stress distribution for Case 2 is similar as shown in Figure 13. The stress values for points $C, D, E, F, G$, and $H$ are shown in Table 5.

In model 3 when the lever makes contact with the surface of the inner side of the lever guide, the stress is greater at points $\mathrm{F}, \mathrm{H}, \mathrm{E}$, and $\mathrm{C}$ on the surface of the inner side and is less at points G, D on the surface of the
Table 5. Stress analysis result for case 2; effect of clearances for the locations of maximum stress.

\begin{tabular}{ccccc}
\hline & \multicolumn{4}{c}{ Maximum stress [MPa] } \\
\cline { 2 - 5 } Point & $\begin{array}{c}\text { Model 3 } \\
\text { (outer side clearance) }\end{array}$ & \multicolumn{2}{c}{ Model 4 } \\
& (inner side clearance) \\
\cline { 2 - 5 } & Mises & Max Principal & Mises & Max Principal \\
\hline C & 202 & 191 & 104 & 111 \\
D & 237 & 200 & 653 & 542 \\
E & 795 & 805 & 621 & 636 \\
F & 159 & 165 & 104 & 113 \\
G & 236 & 195 & 642 & 514 \\
H & 783 & 796 & 583 & 601 \\
\hline
\end{tabular}

outer side than that in Case 1 (clearance $0.3 \mathrm{~mm}$ ). The maximum stress exists at point $\mathrm{E}$. In addition, it can be seen that for model 4 when the lever makes contact with the surface of the outer side of the lever guide, the stress is greater at points $\mathrm{G}$ and $\mathrm{C}$ on the surface of the outer and is less at points $\mathrm{F}, \mathrm{H}, \mathrm{E}$, and $\mathrm{C}$ on the surface of the inner side. The maximum stress is at point $\mathrm{D}$.

Based on the results, the clearance between the lever and lever guide is an important factor in determining the points of maximum stress (Mises stress and maximum principal stress). When the clearance is not taken into account, a countermeasure for those points of stress cannot be considered because the maximum stress occurs on the surface of the inner side. However, even in a fracture test the fracture origin is present on the outer side. This expresses a requirement for considering how the model has deviated from the actual conditions of use, even when using the FEM modeling for fracture analysis.

The calculated results have been introduced into the design of modified vessel and the modified one could demonstrate higher stress to fracture [6]. This experimental result can clearly demonstrate the effectiveness of stress relief grooves we designed.

\section{Conclusions}

The selection of the optimal shape to support the development of high-pressure containers for use in high-pressure processing of food was implemented using finite element analysis. The main results are the follows;

1) When designing a stress relief groove, the lever must overhang the groove $(L \geq 0)$.

2) By introducing a stress relief groove to the $R$ area, maximum stress on the lever guide can be reduced by $10 \%$. This enables the reduction of the maximum stress (Mises stress) to be less than the fatigue strength.

3) The location where maximum stress occurs on the 
lever guide changes in accordance with the clearance between the lever and lever guide. This identified the need to take into account the deviation factor such as design clearance in modeling process.

\section{Acknowledgements}

One of the authors (Y.O.) was partially supported by the Top Runner Incubation System through the AcademiaIndustry Fusion Training in the Promotion of Independent Research Environment for Young Researchers, MEXT, Japan. The authors also appreciate the critical advice of Assoc. Prof. Yukio Miyashita at Nagaoka University of Technology. This research was financially supported by JST project "Development of Technology for Promoting Food Quality".

\section{REFERENCES}

[1] Y. Kishi, "High Pressure Processing Equipment for Food and Biotechnology Fields," Kobe Steel Engineering Report, Vol. 158, No. 2, 2008, p. 25.

[2] Y. Murakami, "Concept of Stress Concentrations," Yokendo Limited, Tokyo, 2005.

[3] Y. Kondo, M. Kubota and S. Kataoka, "Effect of Stress Relief Groove Shape on Fretting Fatigue Strength and Index for the Selection of Groove Shape," Journal of the Society of Materials Science, Vol. 56, No. 12, 2007, pp. 1156-1162. doi: $10.2472 /$ jsms.56.1156

[4] J. Fish and T. Belytschko, "A First Course in Finite Elements," John Wiley \& Sons, New York, 2007. doi:10.1002/9780470510858

[5] D. Nie, Y. Otsuka and Y. Mutoh, "Fatigue Behaviors of Smooth and Notched SUS630 Stainless Steel Specimens", Proceeding of 12th Fractography Symposium, Roskilde, December 2010.

[6] Y. Otsuka, J. Fujii, M. Takato and Y. Mutoh, "Fail-Safe Design by Outer Cover of High Pressure Vessel for Food Processing," Open Journal of Safety Science and Technology, Vol. 1, No. 3, 2011, pp. 89-93. doi:10.4236/ojsst.2011.13009 